\title{
The neurological, psychosocial and demographic correlates of hypergraphia in patients with epilepsy
}

\author{
BRUCE P HERMANN, ${ }^{*} \dagger$ STEVEN WHITMAN, $\ddagger$ ALLEN R WYLER, ${ }^{*} \|$ \\ E T RICHEY,* JADE DELL\$ $\ddagger$
}

From the Regional Epilepsy Center,* Baptist Memorial Hospital, Departments of Psychiatry $\dagger$ and Neurosurgery, $\S$ University of Tennessee-Memphis, Semmes-Murphey Clinic, $\|$ Memphis, TN, Center for Urban Affairs and Policy Research, $\ddagger$ Northwestern University, Evanston, IL, USA

SUMMARY The multiaetiological determinants and correlates of interictal hypergraphia in patients with epilepsy were studied. A variety of neurological, psychosocial and demographic variables were examined in order to determine their relationship to experimental and clinically derived measures of hypergraphia in a sample of 50 patients with epilepsy. The results indicate that hypergraphia is of multifactorial origin in general, with especially strong relationships with measures of psychopathology. These results are related to the larger literature concerned with interictal behavioural change in patients with epilepsy.

Interest in the relationship between epilepsy and hypergraphia (defined as the tendency towards extensive and oftentimes compulsive writing), was initially stimulated by Waxman and Geschwind ${ }^{1-2}$ when they proposed the existence of an interictal syndrome of personality and behavioural change associated with temporal lobe epilepsy. This syndrome was hypothesised to be characterised primarily by alterations in sexual behaviour, increased religiosity, and hypergraphia. Since that publication, a significant research effort has been devoted to the topic of personality and behavioural change in epilepsy, particularly in temporal lobe (or complex partial) epilepsy.

Geschwind's colleagues subsequently elaborated upon the syndrome as a whole suggesting that it consisted of 18 personality and behaviour traits/ characteristics. ${ }^{3}$ Further, specific neuropathogenic mechanisms were proposed, ${ }^{4-7}$ and a variety of empirical studies have been conducted which have attempted to document the existence of the syndrome, its relative specificity to temporal lobe epilepsy, and its underlying determinants (for example references 8 and 9).

Present address and address for reprint requests: Bruce P Hermann, Ph.D., Regional Epilepsy Center, Baptist Memorial Hospital, 899 Madison Ave., Memphis, TN 38146, USA.

Received 31 March 1987 and in revised form 26 August 1987. Accepted 29 August 1987
It should be noted that acceptance of the overall syndrome, its specificity to temporal lobe epilepsy, and agreement as to its possible precursors has met with considerable debate. Some investigators have questioned the specificity of the syndrome to temporal lobe epilepsy in particular and/or epilepsy in general. ${ }^{10-15}$ Others have raised the issue of whether the syndrome is largely secondary to significant psychopathology ${ }^{12-15}$ and, finally, the possibility that other aetiologies (for example, social factors) may be involved has been raised. ${ }^{14}$

In addition to research efforts devoted to the overall syndrome, there has been considerable interest in several individual traits which have been postulated to constitute part of the syndrome. One trait which has received a good deal of attention has been "hypergraphia." This investigation will centre on two major issues associated with hypergraphia. The first concerns the determinants and correlates of the phenomenon. It was argued by Geschwind ${ }^{56}$ that hypergraphia was largely independent of the patient's educational background and intellectual capacity. Further, Geschwind did not believe that hypergraphia was secondary to the psychological stresses associated with epilepsy nor to the anticonvulsant medications. ${ }^{56}$ The primary determinant of the phenomenon was postulated to be the presence of an epileptiform spike focus in the temporal lobe/limbic region. ${ }^{45}$ Despite Geschwind's arguments and his attractive hypothesis, no empirical data have been 
presented which comprehensively evaluate the relative importance of these potential aetiological variables. This is a major aim of the present investigation.

The second issue concerns the assessment and conceptualisation of hypergraphia. Determination of the presence of hypergraphia has rested largely upon relatively informal clinical interview and assessment procedures (for example references 1, 2 and 16). Operational definitions of hypergraphia have not been developed, making it difficult to compare studies. Mungas (personal communication) has recently developed a standardised interview procedure for the clinical assessment of hypergraphia and we will use this procedure in our attempts to identify the correlates of hypergraphia. Other recent investigations have assessed hypergraphia by obtaining patient responses (in writing) to open-ended stimulus questions and have used more specific indices of hypergraphia like response rate and number of words per response. ${ }^{1718}$ At present the relationship between clinical and experimental determinations of hypergraphia is unknown. Further, whether the aetiological variables underlying hypergraphia vary as a function of the method of assessment remains to be determined. Therefore, the purpose of this study is: (1) to investigate systematically the neurological, psychosocial and demographic correlates of hypergraphia, and (2) to contrast clinical and experimental measures of hypergraphia in order to compare the results that are obtained using these different assessment techniques.

\section{Method}

\section{Subjects}

The subjects were referrals to the Baptist Memorial Hospital/University of Tennessee Regional Epilepsy Center. Patients were evaluated for assessment of suitability for focal resection of their epileptogenic lesion, for further diagnostic evaluation because of an unacceptable degree of seizure control, or for reasons concerning differential diagnosis.

In all patients localisation of the primary epileptogenic lesion was attempted. Patients were evaluated via continuous (24-hour) closed circuit TV-EEG monitoring with scalp and or scalp and sphenoidal or nasopharyngeal electrodes. Monitoring was typically carried out until spontaneous seizures were recorded $(82 \%$ of the final sample). In 16 patients further monitoring with subdural strip electrodes ${ }^{19}$ was required in order to identify conclusively the primary seizure focus. The initial subject pool consisted of 58 patients from which eight were subsequently deleted because they had pseudoseizures only $(\mathbf{N}=5)$ or disorders other than epilepsy $(\mathrm{N}=3)$. The final sample therefore consisted of 50 subjects. Their demographic and seizure related characteristics are shown in table 1 .

\section{Procedures}

As part of their evaluation, to all patients complete neu- ropsychological battery was administered. Included in this battery were two measures designed to assess hypergraphia. The first measure was a typed open-ended question which read as follows:

We are very interested in learning about how epilepsy affects our patients' lives. Please begin writing below and tell us, in as much detail as you think important, how epilepsy has affected you and the course of your life, both for the better as well as for the worse. Use as many additional sheets as necessary. Everything you write will be held strictly confidential.

The patient was given two pieces of paper on which to respond and 24-hours elapsed before the reply was collected. If the patient had written nothing after 24 hours, he or she was given another 24 hours in which to respond. If again nothing was written by the patient, the papers were then taken and the response was counted as a zero. Two dependent measures were derived from the patient's writing sample: (1) the total number of words contained in the response, and (2) the presence or absence of references to the meaning or significance of their seizures; philosophical, ethical or religious themes; and unusual writing styles (calligraphy, use of unusual symbols or drawings). These assessments were conducted by an independent rater blinded to all characteristics of each responder. This was done in a fashion identical to that used previously by the present investigators. ${ }^{18}$

In addition to this direct measure of writing behaviour the subjects were clinically evaluated regarding their writing behaviour using an interview-based procedure developed by Mungus (personal communication). This is a Research Diagnostic Criteria-like procedure and is shown in table 2 . This

Table 1 Patient Characteristics $(N=50)$

Age (mean) $(\mathrm{yr})$

$33 \cdot 2$

Education (mean)

$12 \cdot 4$

Age at onset (mean) (yr)

Duration of disorder (mean)

$17 \cdot 0$

Sex

Male

Female

Etiology

Idiopathic

Symptomatic

26

Unclassifiable

Seizure Type

Partial

Simple Partial

Complex Partial

Secondarily Generalised

Primary Generalised

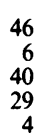

Lateralisation/Localisation of Seizure Onset (by EEG)

Left temporal

Right temporal

Bitemporal (multifocal)

Focal non-temporal

Generalised

Type of EEG Procedures

Scalp

Nasopharyngeal and scalp

Sphenoidal and scalp

Subdural strip electrodes 


\section{Table 2 Clinical interview for hypergraphia}

\section{Definition:}

Tendency to write excessively. Writing goes beyond social, occupational, or educational requirements. Associated with pressure to express self and ideas, perception of having unique thoughts and ideas.

\section{Probing Questions}

Do you write things for yourself or for others, for instance, letters, reports, poetry, a journal or diary, etc.?

When you write, do you do so only for work, business, or school, or do you write when you're not required to?

Do you ever write with the primary purpose of expressing your ideas or opinions? (Do you ever get paid for doing so?)

Do you ever write for your own consumption?

Do you feel that you have unique ideas and opinions that are important to put into writing?

Is writing an important part of your life?

\section{Rating Criteria}

0-No information

1-Writes only when required to by work, business, school.

2-Writes for consumption of others when not required by work, business, or school. Includes letters for purpose of maintaining social contact.

3-Writes for consumption of others. Unsolicited and not reimbursed. Clearly considers self expression an important aspect of writing. Self perception of unique thoughts and ideas.

4-Writes occasionally (twice a week or less) for own consumption (that is, primary motivation is to express self, with limited regard for external consequences). Some pressure to express thoughts and ideas, perception of unique thoughts and ideas.

5-Writes frequently for own consumption. Preoccupied with writing. Writing a central part of life.

clinical evaluation is chacterised by the use of six probe questions. Based on the patient's reponses, the individual is given a hypergraphia rating which ranges from 0 to 5 . For the purposes of this investigation, hypergraphia was defined as a rating of 3,4 , or 5 . Conversely, patients were rated as non-hypergraphic if they received a rating of 0,1 , or 2 . Inter-rater reliability for this system is $\mathbf{0 . 8 7}$.

A primary aim of the present investigation was to determine the multiaetiological correlates of hypergraphia in patients with epilepsy. Therefore, for each of the two measures of hypergraphia, three sets of predictor variables were examined: (1) neurological variables, (2) psychosocial variables, and (3) demographic variables.

Neurological variables included the following: (a) age at onset of the patient's epilepsy; $(b)$ duration of disorder; $(c)$ localisation and lateralisation of seizure onset as revealed by continuous closed circuit TV-EEG monitoring (left temporal lobe, right temporal lobe, bitemporal (multifocal seizure onset), focal non-temporal, generalised); (d) seizure type [partial seizures (simple, complex, and/or secondarily generalised) vs. primary generalised seizures]; and (e) presence vs. absence of multiple seizure types. These variables were determined by a board certified neurosurgeon (ARW) blind to the results of all neuropsychological/behavioural data.

Psychosocial variables assessed several aspects of the patients' lives and included the following: (a) To assess aspects of current emotional status the Depression, Hypomania and Schizophrenia scales of the Minnesota Multiphasic Personality Inventory (MMPI) were utilised. These specific scales were used because it had been our clinical experience that writing behaviour seemed to be associated with the patient's mood state/energy level and degree of unusual thinking. (b) The presence/absence of a history of previous psychiatric treatment (in or out patient) was utilised as a general index of previous emotional disorder of a magnitude requiring professional intervention. (c) General psychosocial adjustment to epilepsy was assessed via the Adjustment to Seizures scale derived from the Washington Psychosocial Seizure Inventory (WPSI). ${ }^{20}(d)$ The number of significant life events which occurred in the past 12 months was assessed via the Life Experiences Survey. ${ }^{21}$ Life event changes are a significant source of stress and it may be the case that patients undergoing more change and turmoil in their lives (which might be attributable in part to their seizure disorder) would be more apt to manifest hypergraphia. (e) Finally, the degree of social support available to the patients was assessed by the Social Support Questionnaire. ${ }^{22}$ Social support might serve as a moderator variable, that is, patients with significant social support might be less apt to manifest hypergraphia as support and counsel would be available thereby negating the need to express oneself via writing activity.

Finally, intellectual/demographic factors included: (a) years of formal education; (b) Verbal, Performance and Full Scale IQ scores derived from the Wechsler Adult Intelligence Scale-Revised; (c) sex; and (d) age.

\section{Data analyses}

First, simple Pearson correlation coefficients were computed between the neurological, psychosocial and demographic predictor variables and, $(a)$ the number of words written in response to the open ended question, as well as $(b)$ a clinical diagnosis of hypergraphia (defined as a rating of 3,4 , or 5 using the Mungas procedure). Next, a stepwise multiple regression analysis was computed with the number of words contained in the written response as the dependent measure, utilising only those predictor variables that showed a significant $(p<0.05)$ simple correlation. Finally, a stepwise multiple logistic regression analysis was computed with the interview based determination of hypergraphia serving as the dependent measure, again using only those predictor variables that showed a significant simple correlation. For both regression analyses, all underlying assumptions were verified.

\section{Results}

Table 3 provides a frequency distribution of the length of the written responses. The mean number of words produced was 318 , with a range of 0 to 3891 . Eightyfour percent wrote less than 400 words while $6 \%$ wrote more than 800 words. These three responses were 1421 words, 1541 words, and 3891 words.

Table 4 shows the distribution of the hypergraphia ratings based upon clinical interview. Using this procedure, $30 \%$ of the sample was classified as hypergraphic. While the proportion of patients classified as hypergraphic differed between the two methods, there 
Table 3 Frequency distribution for length of written responses

\begin{tabular}{lc}
\hline Number of Words & Frequency \\
\hline 0 & 5 \\
$1-100$ & 9 \\
$101-200$ & 19 \\
$201-300$ & 6 \\
$301-400$ & 3 \\
$401-500$ & 0 \\
$501-600$ & 0 \\
$601-700$ & 4 \\
$701-800$ & 1 \\
1421 & 1 \\
1541 & 1 \\
3891 & 1 \\
$\overline{\mathrm{x}}=317.98$ & 5 \\
\hline
\end{tabular}

was a significant correlation between the number of words written and a clinical rating of hypergraphia $(\mathrm{r}=0.47, \mathrm{p}<0.001)$.

Table 5 presents the simple correlations between the predictor and dependent measures. The number of words produced in response to the stimulus was significantly inversely correlated with age at onset $(r=-0.24)$ and the MMPI Depression Scale $(r=-0.32)$ and directly with the duration of disorder $(r=0.26)$, MMPI Hypomania scale $(r=0.32)$, and the number of life events in the past 12 months $(\mathrm{r}=0 \cdot 26)$.

When hypergraphia was defined by clinical interview it was significantly correlated with the presence of primary generalised epilepsy $(r=0.35)$, a previous psychiatric history $(r=0.45)$, increased MMPI Hypomania $(r=0.34)$ and Schizophrenia $(r=0.27)$ scales, increased number of life events $(r=0 \cdot 38)$, and being female $(r=0 \cdot 25)$.

Those variables which showed a significant simple correlation with the dependent measures were entered into a standard regression analysis. For the written responses to the open ended question, two of the variables were identified as making significant independent predictive contributions, the MMPI Hypomania and Depression scales. For the interview based measure of hypergraphia, significant predictors were a history of previous psychiatric treatment and the MMPI Hypomania scale.

Table 4 Frequency distribution of hypergraphia rating based on standardised interview

\begin{tabular}{|c|c|}
\hline Rating & Frequency \\
\hline $\begin{array}{l}1 \\
2\end{array}$ & $28\} 35$ non-hypergraphic \\
\hline $\begin{array}{l}3 \\
4 \\
5\end{array}$ & $\left.\begin{array}{l}3 \\
9 \\
3\end{array}\right\} \quad 15$ hypergraphic \\
\hline
\end{tabular}

*The higher the rating the more writing behaviour manifested by the patient. Ratings of 3 and higher are considered to be indicative of hypergraphia.
Table 5 Pearson correlations between hypergraphia and predictor variables

\begin{tabular}{|c|c|c|}
\hline Predictor variables & Hypergraphia as def & ined by: \\
\hline Neuroepilepsy Factors & Writing Sample & Interview \\
\hline $\begin{array}{l}\text { Age at onset } \\
\text { Duration of disorder } \\
\text { Generalised epilepsy } \\
\text { Laterality of CPS onset: } \\
\text { Left temporal } \\
\text { Right temporal } \\
\text { Multiple seizure types }\end{array}$ & $\begin{aligned} &-0.24(p=0.049)^{1}(p=0.26(p=0.035) \\
& 0.10 \\
& 0.22 \\
&-0 \cdot 17 \\
& 0.14\end{aligned}$ & $\begin{array}{l}-0.14 \\
0.02 \\
0.35(p=0.006) \\
-0.06 \\
-0.14 \\
-0.19\end{array}$ \\
\hline $\begin{array}{l}\text { Psychosocial factors } \\
\text { Previous psychiatric } \\
\text { history } \\
\text { MMPI Depression scale } \\
\text { MMPI Hypomania } \\
\text { scale } \\
\text { MMPI Schizophrenia } \\
\text { scale } \\
\text { WPSI Adjustment to } \\
\text { Seizures scale } \\
\text { Life Experiences Survey } \\
\text { Social Support } \\
\text { Questionnaire }\end{array}$ & $\begin{array}{l}0.02 \\
-0.32(p=0.013) \\
0.32(p=0.013) \\
-0.03 \\
-0.03 \\
0.26(p=0.043) \\
-0.02\end{array}$ & $\begin{array}{l}0.45(p=<0.001) \\
0.05 \\
0.34(p=0.010) \\
0.27(p=0.035) \\
0.16 \\
0.38(p=0.005) \\
0.23\end{array}$ \\
\hline
\end{tabular}

1. Not all variables could be measured for all subjects. Thus, significance levels, which are a function of sample size for these correlation coefficients, vary from variable to variable.

2. Compared with CPS.

Finally, none of the patients manifested an unusual writing style and 10 individuals ( $20 \%$ of sample) produced written responses which contained references to ethical/religious/moral themes, alterations in sexuality, or the meaning/significance of their seizures.

\section{Discussion}

We will review three aspects of our data which relate to the frequency of hypergraphia, its correlates, and the relationship of our findings to previous publications.

While the literature contains several statements concerning the prevalence of hypergraphia in epilepsy, our findings suggest that estimates of the frequency of hypergraphia will vary markedly as a function of the way in which it is measured. Considering first the patients' written responses, it is important to note that operational definitions of hypergraphia have never been developed. For example, how many words are needed before a response is characterised as hypergraphic? In a previous investigation ${ }^{18}$ we found that most written responses fell within a limited range with the exception of a few $(7 \%)$ strikingly longer responses of at least 1,000 words. In the current study, using a different openended question, the mean length of response ( 318 words) closely approximated the average response length in our previous investigation ( 298 words). Further, the current proportion of responses exceeding 1,000 words $(6 \%)$ was virtually identical to our previous findings $(7 \%)$. Given the regularity of findings 
in these two investigations, perhaps it is not unreasonable to suggest that written responses to open-ended questions which exceed 1,000 words are indicative of hypergraphia with a rate of occurrence of approximately $7 \%$ in patients with epilepsy. Comparison with other clinical groups is now needed in order to determine the specificity of hypergraphia to epilepsy relative to other CNS and chronic physical disorders.

Using the clinical interview procedure, $30 \%$ of the sample was classified as hypergraphic, a four-fold increase compared to the written responses. Part of the increase in the interview procedure was due to its assessment of those behaviours not tapped by the writing sample, for example, poems, songs, essays. One might reasonably argue that only clinical ratings of four and greater, or only five, should be defined as hypergraphic. Again, these conceptual difficulties point to the complexities inherent in measuring and defining hypergraphia. It is noteworthy that while rates of hypergraphia varied substantially as a function of the method of assessment, there was a correlation between the clinical and experimental measures of mild to moderate magnitude $(r=0.47$, $p<0.001$ ). Again, we think it is important for future investigations to compare patients with epilepsy with other clinical groups in order to specify the nature and degree of relationship between epilepsy and hypergraphia as defined by this clinical procedure. Additionally, future research effects should be devoted to establishing the validity of the clinical interview and writing sample assessment procedures using hypergraphic and non-hypergraphic patients.

In regard to the aetiology of hypergraphia, our results suggest that the complexity of its underlying factors has been misperceived. For the patients' written responses to the stimulus question the age at onset of epilepsy and MMPI Depression scale were inversely correlated with the number of words written, while duration of epilepsy, the MMPI Hypomania Scale and the number of significant life events occurring during the past year were positively associated with response length (table 5). Because of the substantial intercorrelation which existed among these significant predictor variables, they were entered into a stepwise regression analysis in order to identify those factors which were independently associated with writing behaviour. The results indicated that the only significant independent predictors of length of written response were the patients' affective/mood state. That is, MMPI Depression scores were inversely correlated with response length while MMPI Hypomania scores were positively correlated with response length. The other three variables (duration, age at onset, and number of significant life events) did not explain significant additional proportions of the variance in writing behaviour.
For the interview based measure a clinical rating of hypergraphia was associated with primary generalised epilepsy, increased MMPI Schizophrenia and Hypomania scales, a previous psychiatric history requiring professional intervention, increasing life event changes during the past year, and female sex. Again, because of the intercorrelation among the significant predictor variables, they were placed in a stepwise multiple logistic regression analysis. The results revealed that clinically defined hypergraphia was associated with a history of previous psychiatric treatment, and elevated MMPI Hypomania scale scores. The other variables did not explain significant additional proportions of the variance in writing behaviour.

This association of hypergraphia with general emotional/psychiatric status is consistent with previous investigations which have reported a significant association between psychiatric status and the interictal syndrome of behaviour personality change, ${ }^{12-15}$ although the specific contribution of "affective disorder" noted here is a new observation. Particularly interesting is the significant association of an elevated MMPI Hypomania scale with both measures of hypergraphia. This is perhaps the most striking and most consistent finding which was obtained in this investigation. The presence of an elevated mood state apparently serves to energise writing behaviour and the question arises as to what other behaviours are mobilised by this mood state and why high Ma scales should be noted in epilepsy, a disorder which is generally perceived to be more closely associated with dysphoric mood states. ${ }^{23}$ These questions will need to be addressed in future research efforts.

The role of medication related variables in the aetiology of hypergraphia could not be assessed here because antiepilepsy medications were typically lowered to subtherapeutic levels during the CCTV-EEG monitoring period. It is clear that such factors deserve close scrutiny. ${ }^{12}$

It has been previously suggested that hypergraphia is associated with a spike focus in the right temporal lobe/limbic system. ${ }^{16}$ In our subject pool we found no evidence to support the association of a relationship between hypergraphia and the laterality of the epileptogenic lesion in our patients with CPS of temporal lobe origin. It should be remembered that the majority of our subjects $(82 \%)$ were monitored with scalp, sphenoidal, nasopharyngeal, and/or subdural strip electrodes until spontaneous seizures occurred. The remaining patients had consistently lateralised foci during interictal EEG evaluations. Hence, laterality of lesion was rigourously defined in this investigation.

It is possible that similar multiaetiological inquiries into other traits which compose the purported interictal behavioural syndrome of TLE will reveal similar aetiological complexities. We hypothesise that any 
personality/behavioural changes will be the result of a combination of neurological, psychosocial and medication factors as we have suggested elsewhere. ${ }^{2324}$

\section{References}

i Waxman SG, Geschwind N. The interictal behavior syndrome of temporal lobe epilepsy. Arch Gen Psychiatry 1975;32:1580-6.

2 Waxman SG, Geschwind N. Hypergraphia in temporal lobe epilepsy. Neurology 1974;24:629-36.

3 Bear D, Fedio P. Quantitative analysis of interictal behavior in temporal lobe epilepsy. Arch Neurol 1977;34:454-67.

4 Bear D. Temporal lobe epilepsy: A syndrome of sensory-limbic hyperconnection. Cortex 1979;15:357-84.

5 Geschwind N. Interictal behavioural changes in epilepsy. Epilepsia 1983;23(Suppl.1):523-30.

6 Geschwind N. Pathogenesis of behavior change in temporal lobe epilepsy. In: Ward AA, Penry JK, Purpura D., eds. Epilepsy 1983. New York: Raven Press, 355-70.

7 Bear D, Freeman R, Greenberg M. Behavioral alterations in patients with temporal lobe epilepsy. In: Blumer D., ed. Psychiatric Aspects of Epilepsy, 1985. Washington D.C.: American Psychiatric Press, 197-227.

8 Bear D, Levin K, Blumer D, Chetham D, Ryder J. Interictal behaviour in hospitalised temporal lobe epileptics: Relationship to idiopathic psychiatric syndromes. J Neurol Neurosurg Psychiatry 1982;45:481-8.

9 Bear D, Schenk L, Benson H. Autonomic responses to neutral and emotional stimuli in patients with temporal lobe epilepsy. Am J Psychiatry 1981;138:843-5.

10 Brandt J, Seidman LJ, Kohl D. Personality characteristics of epileptic patients: A controlled study of generalised and temporal lobe cases. J Clin Exp Neuropsychol 1984;7:25-38.

11 Hermann BP, Riel P. Interictal personality and behavioral traits in temporal lobe and generalised epilepsy. Cortex 1981;17:125-8.

12 Rodin E, Schmaltz S. The Bear-Fedio personality inventory and temporal lobe epilepsy. Neurology 1984;34:591-6.
13 Master DR, Toone BK, Scott DF. Interictal behavior in temporal lobe epilepsy. In: Porter RT et al, eds. Advances in Epileptology: XVth International Symposium, 1984. New York: Raven Press, 557-65.

14 Rodin E, Schmaltz S, Twitty G. What does the Bear-Fedio inventory measure? In: Porter RJ et al, eds. Advances in Epileptology: XVth International Symposium, 1984. New York: Raven Press, 551-55.

15 Mungas D. Interictal behavior abnormality in temporal lobe epilepsy: A specific syndrome or non-specific psychopathology? Arch Gen Psychiatry 1982;39:108-11.

16 Roberts JK, Robertson MM, Timble MR. The lateralising significance of hypergraphia in temporal lobe epilepsy. $J$ Neurol Neurosurg Psychiatry 1982;45:131-8.

17 Sachdev HS, Waxman SG. Frequency of hypergraphia in temporal lobe epilepsy: An index of interictal behavior syndrome. J Neurol Neurosurg Psychiatry 1981;44:358-60.

18 Hermann BP, Whitman S, Arnston P. Hypergraphia in epilepsy: Is there a specificity to temporal lobe epilepsy? J Neurol Neurosurg Psychiatry 1983;46:848-53.

19 Wyler AR, Ojemann G, Lettich E, Ward AA. Subdural strip electrodes for localising epileptogenic foci. $J$ Neurosurg 1984;60:1195-200.

20 Dodrill CB, Batzel LW, Queisser HR, Temkin NR. An objective method for the assessment of psychological and social difficulties among epileptics. Epilepsia 1980;21:123-35.

21 Sarason IG, Johnson JH, Siegal JM. Assessing the impact of life changes: Development of the life experiences survey. J Consult Clin Psychology 1978;46:932-46.

22 Sarason IG, Levine HM, Basham RB, Sarason BR. Assessing social support: The Social Support Questionnaire. J Personality Soc Psychology 1983;44:127-39.

23 Hermann BP, Whitman S. Behavioral and personality correlates of epilepsy: A review, methodological critique, and conceptual model. Psychological Bulletin 1984;95:451-97.

24 Hermann BP, Whitman S. Psychopathology in epilepsy: A multietiologic model. In: Whitman S, Hermann BP, eds. Psychopathology in Epilepsy: Social Dimensions, 1986. New York: Oxford University Press, 5-37. 